\title{
An Alternative Approach to the Solution of the Dispersion Relation for a Generalized Lattice Boltzmann Equation
}

\author{
T. Reis and T. N. Phillips \\ School of Mathematics, Cardiff University, \\ Cardiff, CF24 4AG, United Kingdom
}

\begin{abstract}
The simplest and most efficient lattice Boltzmann model that is able to recover the NavierStokes equations is based on a single parameter scattering matrix where the parameter is the first nonzero eigenvalue of the collision matrix. This simple model, based on a single relaxation time, has many shortcomings. Among these is the lack of freedom to extend the model to complex fluids whose stress tensors are characterized by more complicated constitutive relations. The lattice Boltzmann methodology may be generalized by considering the full collision matrix and tuning the matrix elements to obtain the desired macroscopic properties. The generalized hydrodynamics of a generalized lattice Boltzmann equation (LBE) was studied by Lallemand and Luo (Phys. Rev. E 61, 6546 (1999)) by solving the dispersion equation of the linearized LBE. In this paper, an alternative approach to solving the dispersion equation based on a formal perturbation analysis is described. The methodology outlined is systematic, can be readily applied to other lattices and does not require the reciprocals of the relaxation times to be small.
\end{abstract}




\section{INTRODUCTION}

The lattice Boltzmann equation (LBE) is a relatively new numerical technique based on kinetic theory for simulating fluid flow. The LBE evolved from the Lattice Gas Cellular Automata (LGCA) model of Frisch et al. [1], who showed that a simple automaton living on a triangular lattice could provide, in the limit of large-scale motion, a faithful representation of the dynamics of real fluids. The main advantages of LGCA are the absence of round-off error, regular data structures that are ideal for vector processing, local interactions that are ideal for parallel processing and ease of implementation of highly irregular boundary conditions. However, there are some disadvantages, such as statistical noise, exponential complexity of the collision operator with increasing number of states per site and restricted values of the transport coefficients. These disadvantages are due to the Boolean properties of the LGCA model. The issue of statistical noise is characteristic to all particle methods and substantial time/space averaging is required to extract reasonably smooth hydrodynamic signals from LGCA microdynamics. The issue of exponential complexity is also typical of finite-state algorithms. Each time a bit is added the collision rules roughly double in complexity. The LBE, as an alternative description of hydrodynamics, can be viewed as a direct extension of the LGCA developed to overcome the LGCA shortcomings.

The LBE has a mesoscopic character and therefore lies between the classical macroscopic approach to the modelling of fluids based on the Navier-Stokes equations and the microscopic approach based on molecular dynamics. The essential idea behind the method is to model fluid flows by simplified kinetic equations which describe the time evolution of the distribution functions of particles having a discrete set of velocities and moving on a regular lattice. The LBE of McNamara and Zanetti [2] averages the microdynamics before the simulation rather than after it by solving the kinetic equation for the particle distribution instead of tracking the motion of each particle. Therefore, it is less susceptible to noise.

Lallemand and Luo [3] systematically studied the dispersion effects due to the presence of a lattice space by constructing a LBE model in moment space based on the generalized LBE due to d'Humières [4]. An analysis of the generalized hydrodynamics of the model characterized by dispersion, anisotropy and lack of Galilean invariance, for example, facilitates the optimization of the properties of the model through the choice of free parameters associated with a given set of discrete velocities linked to the lattice. The analysis provides 
a mechanism by which comparisons with traditional methods for solving the Navier-Stokes equations can be made. A major advantage of this approach is that the Chapman-Enskog analysis, which is normally used to derive the macroscopic equations from the LBE, becomes redundant. Since the Chapman-Enskog analysis is only applicable when the Knudsen number, which is the ratio of the kinetic to hydrodynamic length scales, is small, the analysis based on the generalized hydrodynamics of the model is more generally valid.

Apart from the work of Lallemand and Luo [3], Behrend et al. [5] have also studied the generalized hydrodynamics of LBE models. The method employed by Lallemand and Luo [3] for solving the dispersion equation of the linearized LBE involves eliminating the offdiagonal terms in the determinant corresponding to the interaction between the conserved and non-conserved moments and expanding in powers of the wave number. This reduces the dispersion equation to a $3 \times 3$ determinant for the conserved modes. The elements of the reduced determinant are then expanded in a series of reciprocals of the relaxation times and the wave number using an appropriate number of terms in order to compute the roots to the required accuracy. This approach requires that the reciprocals of the relaxation times are small in order to perform the expansion.

An alternative, more elegant and transparent approach to the difficult dispersion problem is described in the present paper based on a formal perturbation expansion of the dispersion relation. To our knowledge, the solution of the dispersion equation has only been accomplished, without resorting to numerics, using the approach of Lallemand and Luo [3]. Apart from lacking rigour, there are cases when this approach is unjustified, in particular when the Knudsen number is large. The approach described here is systematic and is readily applied to other lattices. Furthermore, it does not require the reciprocals of the relaxation times to be small in order to perform the analysis. Although it may be true that instabilities in the LBE are triggered more often by boundaries rather than at high Knudsen numbers, our approach shows that the derived transport coefficients (which are identical to those derived by Lallemand and Luo [3]) are valid for all Knudsen numbers.

\section{THE D2Q9 LBE MODEL}

The LBE is characterized by a lattice and some rule describing the manner in which distributions of particles move along lattice directions from one node to another. A simple 
yet sufficiently symmetric and multi-speed lattice is the D2Q9 lattice, shown in Fig. 1. It is a nine velocity model (including a rest particle at the centre) for which an equilibrium solution with all the properties needed to recover the macroscopic hydrodynamic equations can be derived. The nine discrete velocities are given by

$$
\mathbf{c}_{i}= \begin{cases}(0,0), & i=0, \\ (\cos [(\alpha-1) \pi / 2], \sin [(\alpha-1) \pi / 2]), & i=1, \ldots, 4 \\ \sqrt{2}(\cos [(2 \alpha-9) \pi / 4], \sin [(2 \alpha-9) \pi / 4]), & i=5, \ldots, 8\end{cases}
$$

where we have assumed that the unit of velocity is unity.

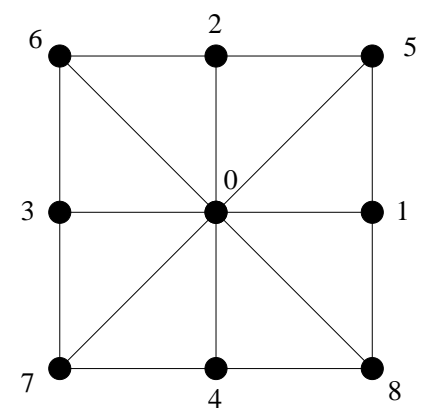

FIG. 1: The D2Q9 lattice.

At each time step, each particle jumps to a neighbouring lattice node and collides with other particles. These advection and collision steps may be described by the equation

$$
N_{i}\left(\mathbf{x}+\mathbf{c}_{i}, t+1\right)=N_{i}(\mathbf{x}, t)+\Omega_{i}(\mathbf{N})
$$

where $\mathbf{N}=\left[N_{0}, \ldots N_{8}\right]^{T}$ and $N_{i}(\mathbf{x}, t), i=0, \ldots, 8$, is a distribution function corresponding to velocity $\mathbf{c}_{i}$ at the lattice point $\mathbf{x}$ at time $t$. This is the so-called lattice Boltzmann equation (LBE). The nine components of $\mathbf{N}$ describe the fluid at each node on the lattice. Once $\mathbf{N}$ is specified at a node on the lattice, the state of the fluid is fully prescribed at this point.

During these collisions the particles scatter but mass and momentum are conserved. In the lattice Boltzmann framework the macroscopic density and momentum are defined by the zeroth and first moments of the distribution function, respectively:

$$
\begin{gathered}
\rho=\sum_{i} N_{i}, \\
\rho \mathbf{u}=\sum_{i} N_{i} \mathbf{c}_{i} .
\end{gathered}
$$


In the LBE approach, the collision operator can be linearised about a local equilibrium distribution function and the elements of the so-called collision matrix may be regarded as a set of free parameters that may be chosen, subject to the conservation constraints, to obtain the desired macroscopic quantities [2].

Higuera and Jimenez [6] conquered the exponential complexity limitation by considering perturbations about the local equilibrium function i.e.

$$
N_{i}=N_{i}^{(e q)}+\epsilon N_{i}^{(1)}+\epsilon^{2} N_{i}^{(2)}+\cdots
$$

where $\epsilon \ll 1$ is the ratio of the microscopic scale to the smallest macroscopic scale. The equilibrium distribution function is required to fulfil the following constraints:

$$
\begin{aligned}
\sum_{i=1}^{b} N_{i}^{(e q)} & =\rho, \\
\sum_{i=1}^{b} N_{i}^{(e q)} \mathbf{c}_{i} & =\rho \mathbf{u} .
\end{aligned}
$$

Now, inserting this form of $N_{i}$ into the collision term and expanding in a Taylor series about $N_{i}^{(e q)}$ gives the quasi-linear lattice Boltzmann equation

$$
N_{i}\left(\mathbf{x}+\mathbf{c}_{i}, t+1\right)-N_{i}(\mathbf{x}, t)=\sum_{j} M_{i j}\left(N_{j}-N_{j}^{(e q)}\right)
$$

where

$$
M_{i j}=\frac{\partial \Omega_{i}\left(\mathbf{N}^{(e q)}\right)}{\partial N_{j}},
$$

defines the collision matrix which determines the scattering rate between directions $i$ and $j$. This matrix is isotropic and cyclic since the scattering depends only on the absolute value of the incoming populations. The importance of this procedure is that it reduces the collision term complexity from $2^{b}$ to $b^{2}$ and then, because of the symmetry of $M_{i j}$, to order $b$ thus making it computationally feasible to perform lattice Boltzmann simulations in three dimensions.

We note that the elements, $M_{i j}$, of the collision matrix, $\mathbf{M}$, are parameters that can be chosen so that $\mathbf{M}$ satisfies the conservation laws and is compatible with the symmetry of the model. These elements are expressed in terms of the non-zero eigenvalues of $\mathbf{M}$ and the spectral analysis of the scattering matrix yields the mass and momentum quantities 
(associated with the null eigenvalues) and the momentum flux tensor (associated with the first non-zero eigenvalue) as well as faster decaying kinetic modes. From this the exact expression for the fluid viscosity can be obtained. In view of the fact that the momentum flux, which is the slowest non-conserved quantity, needs only the leading non-zero eigenvalue of the collision matrix, the LBE model can be simplified yet further to obtain the so-called Lattice Bhatnagar Gross Krook (LBGK) model:

$$
N_{i}\left(\mathbf{x}+\mathbf{c}_{i}, t+1\right)-N_{i}(\mathbf{x}, t)=\omega\left[N_{i}^{(e)}(\mathbf{x}, t)-N_{i}(\mathbf{x}, t)\right]
$$

where $\omega$, which is the first non-zero eigenvalue of $\mathbf{M}$, is a relaxation parameter. This is the simplest and most efficient lattice Boltzmann model that recovers the Navier-Stokes equations.

The equilibrium distribution for the D2Q9 LBGK model is usually taken to be

$$
N_{i}^{(e q)}=\rho w_{i}\left[1+3 \mathbf{c}_{i} \mathbf{u}-\frac{3}{2} u^{2}+\frac{9}{2}\left(\mathbf{c}_{i} \cdot \mathbf{u}\right)^{2}\right]
$$

where $w_{0}=4 / 9, w_{i}=1 / 9(i=1, \ldots, 4)$, and $w_{i}=1 / 36(i=5, \ldots, 8)$. Note that the pressure, defined by $P_{0}=\rho / 3$, satisfies an ideal equation of state. The factor $1 / 3$ is the square of the speed of sound, $c_{s}^{2}$.

The D2Q9 LBGK model is isotropic up to fourth order. A tensor is said to be isotropic if it is invariant under arbitrary rotations and reflections. Isotropic tensors associated with a lattice are constructed from the link vectors $\mathbf{c}_{i}$ by summing over all lattice directions $i$, weighted by a coefficient $w_{i}$. Fourth order isotropy requires

$$
\begin{aligned}
\sum_{i} w_{i} & =1 \\
\sum_{i} w_{i} c_{i \alpha} & =0 \\
\sum_{i} w_{i} c_{i \alpha} c_{i \beta} & =\lambda_{1} \delta_{\alpha \beta}, \\
\sum_{i} w_{i} c_{i \alpha} c_{i \beta} c_{i \gamma} & =0 \\
\sum_{i} w_{i} c_{i \alpha} c_{i \beta} c_{i \gamma} c_{i \delta} & =\lambda_{2}\left(\delta_{\alpha \beta} \delta_{\gamma \delta}+\delta_{\alpha \gamma} \delta_{\beta \delta}+\delta_{\alpha \delta} \delta_{\beta \gamma}\right),
\end{aligned}
$$

where $\lambda_{1}$ and $\lambda_{2}$ are constants, $\delta_{\alpha \beta}$ is the Kronecker delta function and the Greek indices refer to the Cartesian coordinates. For the D2Q9 lattice, we have $\lambda_{1}=1 / 3$ and $\lambda_{2}=1 / 9$. 
The viscosity that appears in the Navier-Stokes equations obtained from this model is proportional to $1 / \omega-1 / 2$. To increase the Reynolds number by lowering the viscosity, one can 'over-relax' the collision operator by using values of $\omega$ in the range $(1,2)$. The method is guaranteed to be numerically stable for $\omega \leq 1$. However, no such guarantees apply when $\omega \geq 1$, and the method can suffer from numerical instabilities, which can limit the highest Reynolds numbers attainable.

The generalized LBE, which is based on multiple relaxation times, leads to improved stability compared with single relaxation time LBGK models [7]. However, alternative approaches have been developed to overcome the stability issue. These include entropic lattice Boltzmann models [8-10], which have been shown to be nonlinearly numerical stable [11]. These models are motivated by the fact that the loss of numerical stability is due to the absence of an $H$-theorem [10]. The idea behind these models is to specify an $H$ function, rather than just the form of the equilibrium distribution. The methodology allows for arbitrarily low viscosity together with a rigorous discrete-time $H$-theorem, and thus absolute stability. The upper limit to the Reynolds numbers attainable by the model is therefore determined by loss of resolution of the smallest eddies, rather than by loss of stability [10].

\section{GENERALIZED LBE}

Although simple and elegant the lattice LBGK equation (10) is not without its shortcomings. The single relaxation parameter, $\omega$, implies that heat transfer takes place at the same rate as momentum transfer. Therefore, the Prandtl number, which is defined to be $\operatorname{Pr}=\nu / \alpha$, where $\alpha$ is the thermal diffusivity, is always of unit value making equation (10) appropriate for isothermal flows only. In addition, the ratio between the bulk and shear viscosities is fixed for LBGK models causing difficulties in simulating flows at high Reynolds number and there is scant freedom to extend the model to complex fluids whose stress tensors are characterised by more complicated constitutive relations. These problems can be addressed by considering the full collision operator in equation (8) and 'tuning' the matrix elements to obtain the desired properties. This task was systematically studied by Lallemand and Luo [3] and in this section we adopt their strategy.

Although we are using the D2Q9 lattice here, the application to other lattices follows a 
similar procedure. Equation (8) can be written in the following concise form:

$$
\left|\delta N\left(\mathbf{x}+\mathbf{c}_{i}, t+1\right)\right\rangle=|\delta N(\mathbf{x}, t)\rangle+\mathbf{M}|\delta N(\mathbf{x}, t)\rangle
$$

where $|X\rangle=\left(X_{0}, X_{1}, \ldots, X_{8}\right)^{\dagger}$ with the superscript $\dagger$ denoting the matrix transpose, and $|\delta N\rangle$ is the fluctuating (non-equilibrium) part of the distribution function. Most D2Q9 models are constructed in a 9-dimensional vector space $\mathbf{R}^{9}$ spanned by $|N\rangle$ but the generalised LBE of Lallemand and Luo is instead based upon the moments $\left\{m_{k} \mid k=0, \ldots, 8\right\}$ of $N_{i}$ which are defined as

$$
m_{k}=\left\langle\psi_{k} \mid N\right\rangle=\left\langle N \mid \psi_{k}\right\rangle, \quad\langle N|=\left(N_{0}, \ldots, N_{8}\right)
$$

where $\left\{\left|\psi_{k}\right\rangle\right\}$ is an orthogonal dual basis set obtained by the Gram-Schmidt procedure from polynomials of the lattice vectors $c_{i \alpha}$. If the members of the basis $\left\{\left|\psi_{k}\right\rangle\right\}$ are also set to be the eigenvectors of the matrix $\mathbf{M}$, the linear relaxation in moment space naturally accomplishes the collision process [7]. To ensure that the correct expressions for density, momentum and stress are obtained, the following vectors are used:

$$
\begin{aligned}
\left|\psi_{0}\right\rangle_{i} & =\left|\mathbf{c}_{i}\right|^{0}=1, \\
\left|\psi_{1}\right\rangle_{i} & =c_{i x}, \\
\left|\psi_{2}\right\rangle_{i} & =c_{i y}, \\
\left|\psi_{3}\right\rangle_{i} & =c_{i x}^{2}-c_{i y}^{2}, \\
\left|\psi_{4}\right\rangle_{i} & =c_{i x} c_{i y} .
\end{aligned}
$$

The corresponding moments $m_{k}, k=0, \ldots, 4$, give the density, $x$-component of momentum, $y$-component of momentum, and the diagonal and off diagonal components of the stress tensor, respectively. The remaining moments can be chosen according to the meso/macroscopic phenomena that are being modelled, such as multi-phase effects [12] and viscoelastic effects [13], for example. Lallemand and Luo [3] find the following remaining (kinetic) moments:

$$
\begin{aligned}
\left|\psi_{5}\right\rangle_{i} & =-4\left|\mathbf{c}_{i}\right|^{0}+3\left(c_{i x}^{2}+c_{i y}^{2}\right) \\
\left|\psi_{6}\right\rangle_{i} & =4\left|\mathbf{c}_{i}\right|^{0}-\frac{21}{2}\left(c_{i x}^{2}+c_{i y}^{2}\right)+\frac{9}{2}\left(c_{i x}^{2}+c_{i y}^{2}\right)^{2} \\
\left|\psi_{7}\right\rangle_{i} & =\left[-5\left|\mathbf{c}_{i}\right|^{0}+3\left(c_{i x}^{2}+c_{i y}^{2}\right)\right] c_{i x} \\
\left|\psi_{8}\right\rangle_{i} & =\left[-5\left|\mathbf{c}_{i}\right|^{0}+3\left(c_{i x}^{2}+c_{i y}^{2}\right)\right] c_{i y}
\end{aligned}
$$


corresponding to the physical quantities of energy, energy square, and the $x$ and $y$ components of the energy flux so that

$$
|m\rangle=\left(\rho, j_{x}, j_{y}, P_{x x}, P_{y y}, e, \epsilon, q_{x}, q_{y}\right)^{\dagger}
$$

Note that to simplify the algebra, the eigenvectors $\left|\psi_{k}\right\rangle$ have not been normalised. Each nonconserved moment has its own equilibrium state which is a function of the conserved moments. A possible choice for these states is [3]:

$$
\begin{aligned}
\psi_{3}^{(e)} & =\alpha_{1} \frac{1}{\left\langle\psi_{4} \mid \psi_{4}\right\rangle}\left[\left\langle\psi_{1} \mid \psi_{1}\right\rangle j_{x}^{2}-\left\langle\psi_{2} \mid \psi_{2}\right\rangle j_{y}^{2}\right) \\
& =\frac{3}{2} \alpha_{1}\left(j_{x}^{2}-j_{y}^{2}\right), \\
\psi_{4}^{(e)} & =\alpha_{2} \frac{\sqrt{\left\langle\psi_{1} \mid \psi_{1}\right\rangle\left\langle\psi_{2} \mid \psi_{2}\right\rangle}}{\left\langle\psi_{5} \mid \psi_{5}\right\rangle}\left(j_{x} j_{y}\right) \\
& =\frac{3}{2} \alpha_{2}\left(j_{x} j_{y}\right), \\
\psi_{5}^{(e)} & =\frac{1}{\left\langle\psi_{6} \mid \psi_{6}\right\rangle}\left[\beta_{1}\left\langle\psi_{0} \mid \psi_{0}\right\rangle \rho+\alpha_{3}\left(\left\langle\psi_{1} \mid \psi_{1}\right\rangle j_{x}^{2}+\left\langle\psi_{2} \mid \psi_{2}\right\rangle j_{y}^{2}\right)\right] \\
& =\frac{1}{4} \beta_{1} \rho+\frac{1}{6} \alpha_{3}\left(j_{x}^{2}+j_{y}^{2}\right), \\
\psi_{6}^{(e)} & =\frac{1}{\left\langle\psi_{7} \mid \psi_{7}\right\rangle}\left[\beta_{2}\left\langle\psi_{0} \mid \psi_{0}\right\rangle \rho+\alpha_{4}\left(\left\langle\psi_{1} \mid \psi_{1}\right\rangle j_{x}^{2}+\left\langle\psi_{2} \mid \psi_{2}\right\rangle j_{y}^{2}\right)\right] \\
& =\frac{1}{4} \beta_{2} \rho+\frac{1}{6} \alpha_{4}\left(j_{x}^{2}+j_{y}^{2}\right), \\
\psi_{7}^{(e)} & =\frac{\left\langle\psi_{1} \mid \psi_{1}\right\rangle}{\left\langle\psi_{7} \mid \psi_{7}\right\rangle} \gamma_{1} j_{x} \\
& =\frac{1}{2} \gamma_{1} j_{x}, \\
\psi_{8}^{(e)} & =\frac{\left\langle\psi_{2} \mid \psi_{2}\right\rangle}{\left\langle\psi_{8} \mid \psi_{8}\right\rangle} \gamma_{1} j_{y} \\
& =\frac{1}{2} \gamma_{3} j_{y},
\end{aligned}
$$

where $\alpha_{1,2,3,4}, \beta_{1,2}$ and $\gamma_{1}$ are constants that will be determined in the next section. Based on the assumption that the non-conserved modes relax linearly towards their equilibrium 
state, the post-collision moments $\hat{m}_{k}$ are given by

$$
\begin{aligned}
\hat{P}_{x x} & =P_{x x}-\tau_{1}\left[P_{x x}-P_{x x}^{(e)}\right], \\
\hat{P}_{x y} & =P_{x y}-\tau_{2}\left[P_{x y}-P_{x y}^{(e)}\right], \\
\hat{e} & =e-\tau_{3}\left[e-e^{(e)}\right], \\
\hat{\epsilon} & =\epsilon-\tau_{4}\left[\epsilon-\epsilon^{(e)}\right], \\
\hat{q}_{x} & =q_{x}-\tau_{5}\left[q_{x}-q_{x}^{(e)}\right], \\
\hat{q}_{y} & =q_{y}-\tau_{6}\left[q_{y}-q_{y}^{(e)}\right],
\end{aligned}
$$

where $\tau_{1}, \ldots, \tau_{6}$, are the relaxation parameters.

There exists a simple linear mapping, $\mathbf{T}$, that relates the distribution functions $|N\rangle$ to the moments $|m\rangle:|m\rangle=\mathbf{T}|N\rangle$ and $|N\rangle=\mathbf{T}^{-1}|m\rangle$ and the linearised lattice Boltzmann equation (17) may be written as

$$
\left|\delta N\left(\mathbf{x}+\mathbf{c}_{i}, t+1\right)\right\rangle=|\delta N(\mathbf{x}, t)\rangle+\mathbf{T}^{-1} \mathbf{C T}|\delta N(\mathbf{x}, t)\rangle,
$$

where

$$
C_{j i}=\left.\frac{\left\langle m_{j} \mid m_{j}\right\rangle}{\left\langle m_{i} \mid m_{i}\right\rangle} \frac{\partial \hat{m}_{i}}{\partial m_{j}}\right|_{|m\rangle=\left|m^{(e)}\right\rangle}
$$

is the collision operator in moment space and is found to be

$$
\mathbf{C}=\left(\begin{array}{ccccccccc}
0 & 0 & 0 & 0 & 0 & 0 & 0 & 0 & 0 \\
0 & 0 & 0 & 0 & 0 & 0 & 0 & 0 & 0 \\
0 & 0 & 0 & 0 & 0 & 0 & 0 & 0 & 0 \\
0 & 3 \tau_{1} \alpha_{1} V_{x} & -3 \tau_{1} \alpha_{1} V_{y} & -\tau_{1} & 0 & 0 & 0 & 0 & 0 \\
0 & 3 \tau_{2} \alpha_{2} V_{y} / 2 & 3 \tau_{2} \alpha_{2} V_{x} / 2 & 0 & -\tau_{2} & 0 & 0 & 0 & 0 \\
\tau_{3} \beta_{1} / 3 & \tau_{3} \alpha_{3} V_{x} / 3 & \tau_{3} \alpha_{3} V_{y} / 3 & 0 & 0 & -\tau_{3} & 0 & 0 & 0 \\
\tau_{4} \beta_{2} / 4 & \tau_{4} \alpha_{4} V_{x} / 3 & \tau_{4} \alpha_{4} V_{y} / 3 & 0 & 0 & 0 & -\tau_{4} & 0 & 0 \\
0 & \tau_{5} \gamma_{1} / 2 & 0 & 0 & 0 & 0 & 0 & -\tau_{5} & 0 \\
0 & 0 & \tau_{6} \gamma_{1} / 2 & 0 & 0 & 0 & 0 & 0 & -\tau_{6}
\end{array}\right)
$$

Clearly, $\mathbf{T}=\left(\left|\psi_{0}\right\rangle, \ldots,\left|\psi_{8}\right\rangle\right)^{t}$.

To examine how the transport coefficients depend on a wave vector $\mathbf{k}$ and to determine the LBE stability properties a Fourier transform is performed on equation (41) which yields:

$$
\mathbf{A}\left|\delta N\left(\mathbf{k}+\mathbf{c}_{i}, t+1\right)\right\rangle=\left[\mathbf{I}+\mathbf{T}^{-1} \mathbf{C T}\right]|\delta N(\mathbf{k}, t)\rangle,
$$


where $\mathbf{I}$ denotes the identity matrix,

$$
A_{i j}=\exp \left(\imath \mathbf{c}_{i} \cdot \mathbf{k}\right) \delta_{i j}
$$

is the streaming operator and $\imath=\sqrt{-1}$. A more concise form of (44) is

$$
|\delta N(\mathbf{k}, t+1)\rangle=\mathbf{L} \mid \delta N((\mathbf{k}, t)\rangle
$$

where

$$
\mathbf{L}=\mathbf{A}^{-1}\left[\mathbf{I}+\mathbf{T}^{-1} \mathbf{C T}\right]
$$

is the linearised evolution operator.

The difference equation (46) has solutions of the form

$$
|G(\mathbf{x}, t)\rangle=\lambda^{t} K_{x}^{m} K_{y}^{n}|X\rangle
$$

where $t$ here denotes time and $m$ and $n$ are indices for space $(\mathbf{x}=m \overline{\mathbf{x}}+n \overline{\mathbf{y}}$ and $\overline{\mathbf{x}}$ and $\overline{\mathbf{y}}$ are unit vectors in Cartesian coordinates). $|X\rangle$ is the initial state. For a fully periodic system the above solution can be chosen as

$$
|\delta N\rangle=\exp (\lambda t-\imath \mathbf{k} \cdot \mathbf{x})|G\rangle
$$

which leads to the following eigenvalue problem [3]:

$$
\lambda|X\rangle=\mathbf{L}|X\rangle
$$

where $\lambda$ satisfies the dispersion relation

$$
\operatorname{det}[\mathbf{I}-\lambda \mathbf{L}]=0
$$

\section{A. Perturbation Expansion of the Dispersion Relation}

The roots of the dispersion relation (51) determine the transport coefficients and their dependence on $\mathbf{k}$. The solution of the above equation also provides the solution of the initial value problem (46):

$$
|\delta N(\mathbf{k}, t+1)\rangle=\mathbf{L}^{t}|\delta N(\mathbf{k}, 0)\rangle=\sum_{\mu} \lambda_{\mu}^{t}\left|\phi_{\mu}\right\rangle\left\langle\phi_{\mu}^{\prime} \mid \delta N(\mathbf{k}, 0)\right\rangle
$$


where $\left|\phi_{\mu}\right\rangle$ is the right eigenvector of $\mathbf{L}$ corresponding to eigenvalue $\lambda_{\mu}$ and $\left\langle\phi_{\mu}^{\prime}\right|$ is the left eigenvector. Note that since the matrix $\mathbf{L}$ is non-symmetric and non-Hermitian, the right and left eigenvectors are neither equal nor orthogonal, but they do form a complete bi-orthonormal set, i.e.

$$
\begin{aligned}
\sum_{\mu}\left|\phi_{\mu}\right\rangle\left\langle\phi_{\mu}^{\prime}\right| & =\mathbf{I}, \\
\left\langle\phi_{v}^{\prime} \mid \phi_{\mu}\right\rangle & =\delta_{v \mu} .
\end{aligned}
$$

The dispersion relation is complicated and one cannot get analytic expressions for the roots in $z$, except for some very special cases. When $k_{x}=k_{y}=0$ equation (51) factorises as

$$
\begin{gathered}
(1-\lambda)^{3}\left[\lambda-\left(1-\tau_{1}\right)\right]\left[\lambda-\left(1-\tau_{2}\right)\right]\left[\lambda-\left(1-\tau_{3}\right)\right] \times \\
{\left[\lambda-\left(1-\tau_{4}\right)\right]\left[\lambda-\left(1-\tau_{5}\right)\right]\left[\lambda-\left(1-\tau_{6}\right)\right]=0}
\end{gathered}
$$

and since the hydrodynamic regime corresponds to long times and large spacial scales (i.e. when $k=|\mathbf{k}| \rightarrow 0$ ), the hydrodynamic eigenvalues are those with $z$ close to 1 . Luo and Lallemand [3] suggest solving equation (50) by expanding the evolution operator, $\mathbf{L}$, in powers of $k$ and applying Gaussian elimination to the matrix using $1 / \tau_{\alpha}$ as small parameters for the non-conserved (kinetic) modes. Doing this successfully leads to a new $3 \times 3$ determinant for the conserved (hydrodynamic) modes. Due to the complexity of the dispersion matrix and the large number of unknowns this procedure is rather cumbersome. Therefore, we choose to solve the dispersion equation by considering a perturbation expansion of equation (50) $[14]$, i.e.

$$
\begin{aligned}
\mathbf{L} & =\mathbf{L}^{(0)}+\mathbf{L}^{(1)}+\mathbf{L}^{(2)}+\ldots, \\
\phi_{\mu} & =\phi_{\mu}^{(0)}+\phi_{\mu}^{(1)}+\phi_{\mu}^{(2)}+\ldots, \\
z_{\mu} & =\lambda_{\mu}^{(0)}+\lambda_{\mu}^{(1)}+\lambda_{\mu}^{(2)}+\ldots
\end{aligned}
$$

where the superscripts refer to the order of $k \ll 1$ and

$$
\begin{aligned}
\mathbf{L}^{(n)} & =\mathbf{K}^{(n)}\left[\mathbf{I}+\mathbf{M}^{-1} \mathbf{C M}\right] \\
K_{i j}^{n} & =\frac{1}{n !}\left(-\imath \mathbf{k} \cdot \mathbf{c}_{i}\right)^{n} \delta_{i j} .
\end{aligned}
$$


The transport coefficients are related to the eigenvalues of $\mathbf{L}$ through the following [3]:

$$
\begin{aligned}
\nu(\mathbf{k}) & =-\frac{1}{k^{2}} \Re\left(\ln \lambda_{T}(\mathbf{k})\right), \\
g(\mathbf{k}) V \cos \psi & =-\frac{1}{k} \Im\left(\ln \lambda_{T}(\mathbf{k})\right), \\
\frac{1}{2} \nu(\mathbf{k})+\zeta(\mathbf{k}) & =-\frac{1}{k^{2}} \Re\left(\ln \lambda_{ \pm}(\mathbf{k})\right), \\
c_{s}(\mathbf{k}) \pm g(\mathbf{k}) V \cos \psi & =\mp \frac{1}{k} \Im\left(\ln \lambda_{ \pm}(\mathbf{k})\right),
\end{aligned}
$$

where $\nu$ is the kinematic viscosity, $\zeta$ is the bulk viscosity, $g$ is a Galilean invariant factor ( $g=1$ implies Galilean invariance), $\psi$ is the angle between $\mathbf{V}$ and $\mathbf{k}$, and $\lambda_{T}$ and $\lambda_{ \pm}$are the eigenvalues corresponding to the tranverse and longitudinal hydrodynamic modes of the system. The transport coefficients are to be obtained through a perturbation analysis so the following series expansion is used:

$$
\begin{aligned}
\nu(\mathbf{k}) & =\nu^{(0)}+\nu^{(1)} k^{2}+\ldots+\nu^{(n)} k^{2 n}+\ldots \\
\zeta(\mathbf{k}) & =\zeta^{(0)}+\zeta^{(1)} k^{2}+\ldots+\zeta^{(n)} k^{2 n}+\ldots \\
C(\mathbf{k}) & =C^{(0)}+C^{(1)} k^{2}+\ldots+C^{(n)} k^{2 n}+\ldots \\
g(\mathbf{k}) & =g^{(0)}+g^{(1)} k^{2}+\ldots+g^{(n)} k^{2 n}+\ldots
\end{aligned}
$$

Examining the resulting dispersion equation at different orders of $k$ gives the transport coefficients and the bounds on the free parameters.

\section{B. The Eigenvalues and Transport Coefficients}

Substituting the expansions (57), (58) and (59) into the dispersion equation (50) yields the set of equations:

$$
\begin{aligned}
\mathbf{L}^{(0)}\left|\phi_{\mu}^{(0)}\right\rangle & =\lambda_{\mu}^{(0)}\left|\phi_{\mu}^{(0)}\right\rangle \\
\left(\mathbf{L}^{(0)}-\lambda_{\mu}^{(0)} \mathbf{I}\right)\left|\phi_{\mu}^{(1)}\right\rangle & =-\left(\mathbf{L}^{(1)}-\lambda_{\mu}^{(1)} \mathbf{I}\right)\left|\phi_{\mu}^{(0)}\right\rangle \\
\left(\mathbf{L}^{(0)}-\lambda_{\mu}^{(0)} \mathbf{I}\right)\left|\phi_{\mu}^{(2)}\right\rangle & =-\left(\mathbf{L}^{(2)}-\lambda_{\mu}^{(2)} \mathbf{I}\right)\left|\phi_{\mu}^{(0)}\right\rangle-\left(\mathbf{L}^{(1)}-\lambda_{\mu}^{(1)} \mathbf{I}\right)\left|\phi_{\mu}^{(1)}\right\rangle .
\end{aligned}
$$

We first consider the simpler case in which the streaming velocity $\mathbf{V}=\mathbf{0}$. Equation (70) is easily solved and an eigenvalue $\lambda_{\mu}^{(0)}=1$ with a three fold degeneracy is found. These eigenvalues correspond to three hydrodynamic (conserved) modes. Due to the degeneracy 
of $\lambda_{\mu}^{(0)}$, the corresponding $O(1)$ eigenvectors, $\left|z_{n}\right\rangle$, are linearly dependent and the general solution to equation (70) is a linear combination of these vectors:

$$
\left|\phi_{\mu}^{(0)}\right\rangle=\sum_{n} B_{\mu, n}\left|z_{n}\right\rangle
$$

where the $B_{\mu, n}$ 's are coefficients to be determined.

To solve the order $k$ equation we multiply (71) to the left with $\left\langle z_{m}^{\prime}\right|$ :

$$
\left\langle z_{m}^{\prime}\left|\mathbf{L}-\lambda_{\mu}^{(1)} \mathbf{I}\right| \phi_{\mu}^{(0)}\right\rangle=\sum_{n}\left\langle z_{m}^{\prime}\left|\mathbf{L}-\lambda_{\mu}^{(1)} \mathbf{I}\right| z_{n}\right\rangle B_{\mu, n}=0
$$

where the left vectors, $\left\langle z_{m}^{\prime}\right|$, are found by solving the transpose of equation (70). For future reference we define the general solution to the 'left' equation to be

$$
\left\langle\phi_{v}^{(0)}\right|=\sum_{m} B_{v, m}^{\prime}\left\langle z_{m}^{\prime}\right|
$$

Equation (74) is an eigenvalue problem to order $k$ in the sub-space spanned by the hydrodynamic modes, i.e. $\operatorname{det}\left[\mathbf{L}-\lambda_{\mu}^{(1)} \mathbf{I}\right]=0$. The characteristic polynomial is of degree 3 in $\lambda_{\mu}^{(1)}$ and can be solved to find

$$
\begin{aligned}
& \lambda_{T}^{(1)}=0, \\
& \lambda_{ \pm}^{(1)}= \pm \imath k c_{s},
\end{aligned}
$$

with the speed of sound squared given by

$$
c_{s}^{2}=\frac{1}{3}\left(2+\frac{\beta_{1}}{8}\right)
$$

To ensure positivity of the speed of sound we require $\beta_{1}>-16$. The coefficients $B_{\mu, n}$, and hence the eigenvectors $\left|\phi_{\mu}^{(0)}\right\rangle$, are found by substituting $\lambda_{\mu}^{(1)}$ into equation (74) and solving the linear system. A similar procedure applied to the left equation yields the particular solution $\left\langle\phi_{\mu}^{\prime(0)}\right|$. The general solution to equation (71) is

$$
\left|\phi_{\mu}^{(1)}\right\rangle=-\left(\mathbf{L}^{(0)}-\lambda_{\mu}^{(0)} \mathbf{I}\right)^{-1}\left(\mathbf{L}^{(1)}-\lambda_{\mu}^{(1)} \mathbf{I}\right)\left|\phi_{\mu}^{(0)}\right\rangle+\sum_{n} D_{\mu, n}\left|\phi_{\mu}^{(0)}\right\rangle
$$

where $D_{\mu, n}$ are constant coefficients. Since the matrix $\mathbf{L}^{(0)}-\lambda_{\mu}^{(0)} \mathbf{I}$ is singular we find the first term on the right-hand-side of equation (78), which we call $\left|\sigma_{\mu}^{(1)}\right\rangle$, by applying the method of Gaussian elimination and back-substitution to the linear system (71). 
To find the eigenvalues at order $k^{2}$ we multiply equation (72) to the left by $\left\langle\phi_{v}^{(0)}\right|$ and rearrange to obtain

$$
\begin{gathered}
\lambda_{\mu}^{(2)}=\frac{1}{\left\langle\phi_{v}^{\prime(0)} \mid \phi_{\mu}^{(0)}\right\rangle}\left[\left\langle\phi_{v}^{(0)}\left|\mathbf{L}^{(2)}\right| \phi_{\mu}^{(1)}\right\rangle+\left\langle\phi_{v}^{(0)}\left|\mathbf{L}^{(1)}-\lambda_{\mu}^{(1)} \mathbf{I}\right| \sigma_{\mu}^{(1)}\right\rangle\right. \\
\left.+\sum_{n} D_{\mu, n}\left\langle\phi_{v}^{\prime(0)}\left|\mathbf{L}^{(1)}-\lambda_{\mu}^{(1)} \mathbf{I}\right| \phi_{\mu}^{(0)}\right\rangle\right] .
\end{gathered}
$$

For $v=\mu$ the last term on the right-hand-side of equation (79) vanishes and we find the following expressions for the eigenvalues:

$$
\begin{aligned}
\lambda_{T}^{(2)} & =-k^{2} \nu_{0}=\frac{\left\langle\phi_{T}^{(0)}\left|\mathbf{L}^{(2)}\right| \phi_{T}^{(1)}\right\rangle+\left\langle\phi_{T}^{(0)}\left|\mathbf{L}^{(1)}\right| \sigma_{T}^{(1)}\right\rangle}{\left\langle\phi_{T}^{(0)} \mid \phi_{T}^{(0)}\right\rangle} ; \\
\lambda_{ \pm}^{(2)} & =-k^{2}\left(\nu_{0}+\zeta_{0}\right) \\
& =\frac{\left\langle\phi_{ \pm}^{\prime(0)}\left|\mathbf{L}^{(2)}\right| \phi_{ \pm}^{(1)}\right\rangle+\left\langle\phi_{ \pm}^{(0)}\left|\mathbf{L}^{(1)} \pm \imath k c_{s} \mathbf{I}\right| \sigma_{ \pm}^{(1)}\right\rangle}{\left\langle\phi_{ \pm}^{(0)} \mid \phi_{ \pm}^{(0)}\right\rangle} .
\end{aligned}
$$

The above depend on the direction of the wave vector $\mathbf{k}$. To eliminate this effect and ensure isotropy we require the expressions to factorise in $k^{2}$. This is achieved if we set

$$
\frac{1}{\tau_{2}}-\frac{1}{2}=2\left(\frac{1}{\tau_{1}}-\frac{1}{2}\right) \frac{\gamma_{1}+4}{2-\gamma_{1}}
$$

which leads to expressions for the kinematic and bulk viscosity in the long wave-length limit $(\mathbf{k} \rightarrow \mathbf{0}):$

$$
\begin{aligned}
& \nu_{0}=\frac{2-\gamma_{1}}{12}\left(\frac{1}{\tau_{1}}-\frac{1}{2}\right) \\
& \zeta_{0}=\frac{\gamma_{1}+10-12 c_{s}^{2}}{24}\left(\frac{1}{\tau_{3}}-\frac{1}{2}\right),
\end{aligned}
$$

where $-4<\gamma_{1}<2$ and $0<\tau_{1}, \tau_{2}, \tau_{3}<2$ by the positivity of the transport coefficients.

To determine the other adjustable parameters and narrow the bounds of $\beta_{1}$ and $\gamma_{1}$ we now consider the dispersion equation with a constant streaming velocity V. To satisfy Galilean invariance we must have $g_{0}=1$, which is only obtained when

$$
\alpha_{1}=\alpha_{2}=\frac{2}{3}, \quad \alpha_{3}=18
$$

We proceed in the same way as the case when $\mathbf{V}=\mathbf{0}$ by examining the dispersion equation to different orders in $k$. After solving equation (70), equation (71) is solved to find the speed of sound, $C_{s}$ :

$$
C_{s}=\mathbf{V} \cdot \mathbf{k} \pm \sqrt{c_{s}^{2}+(\mathbf{V} \cdot \mathbf{k})^{2}}
$$


The second order eigenvalues are complicated expressions that depend on the direction of the wave vector $k$. If we set $\gamma_{1}=-2$, the shear and bulk viscosities are found to be

$$
\begin{aligned}
\nu_{0}= & {\left[\tau_{3}\left(2-\tau_{1}\right)\left[c_{s}^{2}+\left(1-3 c_{s}^{2}\right)(\mathbf{V} \cdot \mathbf{k})^{2}\right]+3\left[2\left[\tau_{1}-\tau_{3}\right]\right.\right.} \\
& \left.\left.+\tau_{1}\left(\tau_{3}-2\right)(\mathbf{V} \cdot \mathbf{k})^{2} / V\right]\right] /\left[6 \tau_{3} \tau_{1}\left((\mathbf{V} \cdot \mathbf{k})^{2}+c_{s}^{2}\right)\right] \\
\zeta_{0}= & {\left[( \mathbf { V } \cdot \mathbf { k } ) \sqrt { c _ { s } ^ { 2 } + ( \mathbf { V } \cdot \mathbf { k } ) ^ { 2 } } \left(12 V^{2}\left[\left(\tau_{3}-\tau_{1}\right)+\tau_{3}\left(\tau_{1}-2\right)(\mathbf{V} \cdot \mathbf{k})^{2} / V^{2}\right]\right.\right.} \\
& \left.\left(2 \tau_{3}-3 \tau_{3} \tau_{1}+4 \tau_{1}\right)\left(1-3 c_{s}^{2}\right)\right)+3(\mathbf{V} \cdot \mathbf{k})^{2}\left[(\mathbf{V} \cdot \mathbf{k})^{2}\left(2 \tau_{1}+3 \tau_{1} \tau_{3}-8 \tau_{1}\right)\right. \\
& \left.6 V^{2}\left(\tau_{3}-\tau_{1}\right)\right]+2(\mathbf{V} \cdot \mathbf{k})^{2}\left[6\left(2 \tau_{1} \tau_{3}-\tau_{3}-\tau_{1}\right) c_{s}^{2}+\tau_{1}\left(2-\tau_{3}\right)\right] \\
+ & c_{s}^{2}\left[6 V^{2}\left(\tau_{3}-\tau_{1}\right)+\tau_{1}\left(2-\tau_{3}\right)\right. \\
& \left.\left.\times\left(2-3 c_{s}^{2}\right)\right]\right] /\left[12 \tau_{1} \tau_{3}\left((\mathbf{V} \cdot \mathbf{k})^{2}+c_{s}^{2}\right)\right]
\end{aligned}
$$

The effect of $V$ on the transport coefficients is clear. Setting $c_{s}^{2}=1 / 3$ (that is, $\beta_{1}=-8$ ) eliminates the first order effect of $\mathbf{V}$ on $\zeta_{0}$ and the second order effect on $\nu_{0}$. Lallemand and Luo [3] argue that the second order effects of $\mathbf{V}$ on $C_{s}$ and $\zeta_{0}$ can be removed by considering a more complicated 13 velocity lattice and allowing for compressibility effects in the equilibrium properties. Note that the model reduces to the LBGK equation (10) if we set all the relaxation parameters to be equal $\left(\tau_{\alpha}=\omega\right)$ and choose $\beta_{2}=4, \alpha_{4}=-18$.

\section{CONCLUSIONS}

The generalized hydrodynamics of the a generalized LBE is studied using a transparent and formal perturbation analysis. The value of this approach is it provides a means to analyse the generalised hydrodynamic behaviour of the LBE which can be compared to that of the Navier-Stokes equations. The perturbation analysis obviates the need to perform a Chapman-Enskog analysis, which is only applicable when the Knudsen number is small, to retrieve the macroscopic equations. If (to a certain value of $\mathbf{k}$ ) the modes of the LBE and Navier-Stokes equations behave in exactly the same way (as shown in [3]) then there is no distinction between the two sets of equations (to a given order of $\mathbf{k}$ ) and the ChapmanEnskog analysis (which can be rather cumbersome for models more complicated than the D2Q9 LBGK equation (10)) can be bypassed. The approach described here provides an alternative treatment to generalized hydrodynamics to that presented by Lallemand and Luo [3], which is not restricted to situations where $1 / \tau_{i}, i=1, \ldots, 6$, are small. The expressions 
obtained for the hydrodynamic transport coefficients agree with those in [3] and are optimal in the sense that they yield the desirable properties. Moreover, the methodology described here is systematic and can be readily modified to study any lattice. The perturbation analysis of the dispersion equation for the D2Q9 LBE yields the desired transport coefficients.

The ability to study the LBE for a range of $k$ (which the Chapman-Enskog analysis cannot do) reveals some shortcomings of the D2Q9, namely the dependence of the transport coefficients on the mean velocity $\mathbf{V}$. The generalised LBE separates different time scales within the model, allowing for the incorporation of sophisticated physics such as energy modes (important for defining non-isothermal LBE's that are consistent with thermodynamics) and non-Newtonian constitutive relations for the stress tensor. It has been shown that having separate relaxation times for the kinetic modes can increase the stability of the model [3].

This approach also suggests a means for developing a LBE for viscoelastic fluids by extending the lattice domain (e.g. from D2Q9 to D2Q11) and coupling the eigenvectors associated with the viscous stress tensor to two new eigenvectors that are responsible for memory effects. The coupling is obtained by modifying the original viscous eigenvectors. Therefore, we have the modified eigenvalues of the collision/evolution operator together with two additional ones. To obtain viscoelastic effects it is also important that the different modes relax at different rates, which is achieved though the values of the eigenvalues. In other words, to develop a class of viscoelastic LBE's, the choice of collision matrix/eigenvectors is important.

[1] U. Frisch, B. Hasslacher, and Y. Pomeau, Phys. Rev. Lett. 56, 1505 (1986).

[2] G. McNamara and G. Zanetti, Phys. Rev. Lett. 761, 2332 (1988).

[3] P. Lallemand and L.-S. Luo, Phys. Rev. E 61, 6546 (1999).

[4] D. d'Humières, in Rarefied Gas Dynamics: Theory and Simulations, edited by B. D. Shizgal and D. P. Weaver (AIAA, Washington DC, 1992), vol. 159.

[5] O. Behrend, R. Harris, and P. B. Warren, Phys. Rev. E 50, 4586 (1994).

[6] F. Higuera and J. Jimenez, Europhys. Lett. 9, 663 (1986).

[7] D. d'Humières, I. Ginzburg, M. Krafczyk, P. Lallemand, and L.-S. Luo, Phil. Trans. R. Soc. 
Lond. A 360, 437 (2001).

[8] I. V. Karlin, A. Ferrante, and H. C. Öttinger, Europhys. Lett. 47, 182 (1999).

[9] I. V. Karlin, A. N. Gorban, S. Succi, and V. Boffi, Phys. Rev. Lett. 81, 6 (1998).

[10] B. M. Boghasian, J. Yepez, P. V. Coveney, and A. Wagner, Proc. Roy. Soc. Lond. A 457, 717 (2001).

[11] S. Ansumali, I. V. Karlin, and H. C. Öttinger, Europhys. Lett. 63, 798 (2003).

[12] M. McCraken and J. Abraham, Phys. Rev. E 71, 036701 (2005).

[13] L. Giraud, D. d'Humieres, and P. Lallemand, Int. J. Mod. Phys. C 8, 805 (1997).

[14] J. Rivet and J. Boon, Lattice Gas Hydrodynamics (Cambridge University Press, Cambridge, 2001). 